\title{
Leveraging Nanoscale Plasmonic Modes to Achieve Reproducible Enhancement of Light
}

\author{
Ryan T. Hill, ${ }^{\dagger}$ Jack J. Mock, ${ }^{\ddagger}$, Yaroslav Urzhumov, ${ }^{\ddagger} \S$ David S. Sebba," Steven J. Oldenburg," \\ Shiuan-Yeh Chen, ${ }^{\mp, \perp}$ Anne A. Lazarides, ${ }^{\dagger, \S, \perp}$ Ashutosh Chilkoti, ${ }^{\dagger, \#}$ and David R. Smith ${ }^{*},{ }^{\ddagger},{ }^{\prime}$ \\ ${ }^{\dagger}$ Center for Biologically Inspired Materials and Material Systems (CBIMMS), Duke University, Box 90271, Durham, \\ North Carolina 27708, ${ }^{\star}$ Department of Electrical and Computer Engineering, Duke University, Box 90201 , \\ Durham, North Carolina 27708, ${ }^{\S}$ Center for Metamaterials and Integrated Plasmonics (CMIP), Duke University, \\ Box 90271, Durham, North Carolina 27708, "nanoComposix, Inc., 4878 Ronson Court, Suite K, San Diego, \\ California 92111, ${ }^{\perp}$ Department of Mechanical Engineering and Materials Science, Duke University, Box 90300, Durham, \\ North Carolina 27708, and \# Department of Biomedical Engineering, Duke University, Box 90281, \\ Durham, North Carolina 27708
}

\begin{abstract}
The strongly enhanced and localized optical fields that occur within the gaps between metallic nanostructures can be leveraged for a wide range of functionality in nanophotonic and optical metamaterial applications. Here, we introduce a means of precise control over these nanoscale gaps through the application of a molecular spacer layer that is self-assembled onto a gold film, upon which gold nanoparticles (NPs) are deposited electrostatically. Simulations using a three-dimensional finite element model and measurements from single NPs confirm that the gaps formed by this process, between the NP and the gold film, are highly reproducible transducers of surface-enhanced resonant Raman scattering. With a spacer layer of roughly $1.6 \mathrm{~nm}$, all NPs exhibit a strong Raman signal that decays rapidly as the spacer layer is increased.
\end{abstract}

KEYWORDS Nanophotonics, plasmon resonant nanoparticles, surface-enhanced resonant Raman scattering, gold film, doughnut

1 he investigation of metals for use in guiding and controlling light has accelerated over the past decade, as metals offer a potential route to the nanoscale miniaturization of photonic devices. Though metals exhibit considerably more absorption than do their conventional dielectric counterparts, they nevertheless possess unique properties that can add new capabilities and functionalities to photonic devices. In particular, nanosized metal structures support localized plasmon resonances, or coupled electronic/ optical excitations, whose properties can be controlled by tailoring the size and shape of the NP or through the controlled spacing of several NPS. ${ }^{1-6}$ Incident light can interact with plasmon resonances and be localized to subwavelength volumes, where an enhancement of the local fields over the incident field typically occurs. These enhanced, localized fields can be used to drive nonlinearities in molecular systems $s^{7,8}$ and have been suggested as the basis for optical metamaterials, ${ }^{9,10}$ nanophotonic circuits ${ }^{11}$ and devices such as plasmonic lasers, ${ }^{12-15}$ mixers, ${ }^{16,17}$ and transistors. ${ }^{18}$

The largest field enhancements in plasmon resonant nanostructures coincide with the most tightly confined fields. A NP with sharp asperities or corners, for example, can enhance fields by a factor of 100 or more in the localized, subnanometer volumes surrounding the asperities. ${ }^{19}$ Simi-

* To whom correspondence should be addressed. E-mail: drsmith@ee.duke.edu. Received for review: 07/13/2010

Published on Web: 08/30/2010 larly, fields can also be strongly localized and enhanced within the subnanometer regions between NPs that are spaced within a few nanometers. ${ }^{9,20-23}$ For those geometries that yield large enhancements, the exact characteristics of the localized field pattern and the enhancement ratio will depend crucially on the subnanometer features associated with the NP. The key to unlocking the potential of plasmonic structures is to pattern or control these features at the subnanometer scale. While nanotechnology has provided us with powerful approaches to nanoscale patterning through lithographic and self-assembly techniques, the control over subnanometer feature sizes required to reliably achieve the strongest field enhancements has remained elusive.

A well-known phenomenon associated with plasmonic field-enhancements is that of surface-enhanced Raman scattering (SERS) in which the inelastic scattering from the vibrational modes of a molecule near a plasmon resonant structure is enhanced by many orders of magnitude. It is universally found that the commonly used roughened films and colloidal NP suspensions generate only a small percentage of "hot" spots ${ }^{24,25}$ from which Raman signals can be detected. Thus, while SERS can be easily demonstrated in bulk solutions and substrates, chip-scale devices or even nanosized SERS-based sensors have been impractical to implement due to the typically low yield in generating the SERS active nanostructure.

The search for improved and consistently reliable SERS substrates with uniformly large field enhancements has 

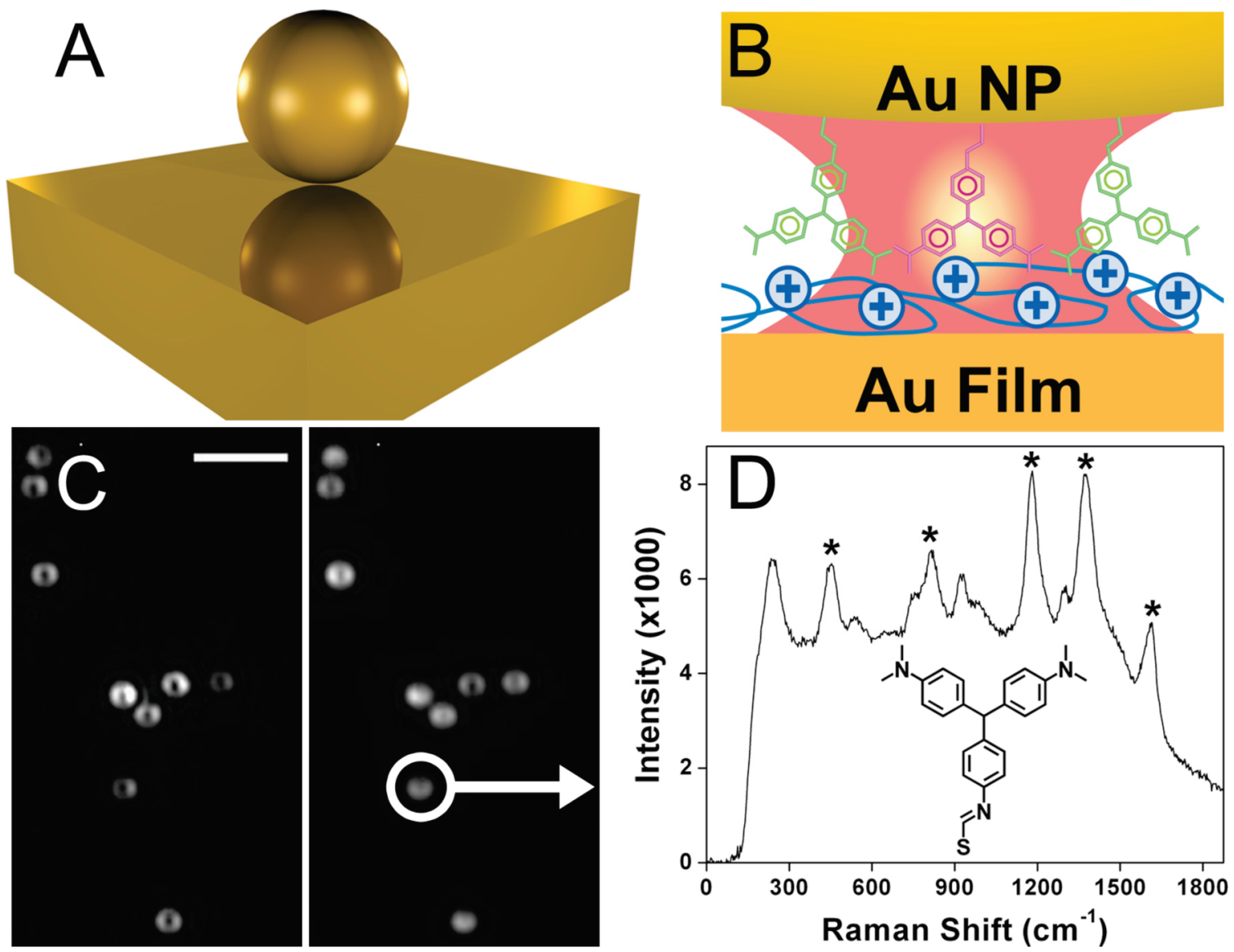

FIGURE 1. A gold nanoparticle (NP) near a gold film (A) can be represented as a plasmonically coupled "dimer" formed between the real NP and its image. (B) Localized NP-film field enhancement is used to drive SERRS from MGITC molecules adsorbed on the surface of the NP and within the NP-film gaps, which were created by a single molecular layer of PAH $(0.6 \mathrm{~nm}$ layer thickness). Approximately $1-5 \mathrm{MGITC}$ molecules are estimated to fit into the enhanced region and contribute to SERRS. ${ }^{38}$ (C, left) Single NP-film/MGITC resonators elastically scatter TIR incident $633 \mathrm{~nm}$ light from a HeNe laser (scale bar $=3 \mu \mathrm{m}$ ). (C, right) SERRS from the same field was collected by blocking the laser scatter with a long pass filter and was measurable from every single film-coupled NP (100\% yield). (D) Representative SERRS spectrum collected from a single NP-film gap. The marked lines at 448, 807, 1180,1370, 1618 relative $\mathrm{cm}^{-1}$ are used to identify MGITC. ${ }^{40}$

resulted in both the fabrication of asymmetric NPs as well as the controlled formation of NP clusters. In the latter case, structures have been created by a number of techniques, including patterning by electron beam or by nanosphere lithography, ${ }^{26-28}$ assembly of plasmonic nanostructures in solution using biochemical linkages, ${ }^{29-31}$ or control of the relative position of plasmonic nanostructures using atomic force microscopy. ${ }^{32}$ Measurements from samples produced by all of these techniques have confirmed the sensitivity of the field enhancement to the dimensions of the gap between the coupled plasmonic surfaces. Yet, none of these techniques has to date provided sufficient control over the gap dimensions to render any of the fabricated substrates robust enough to reliably support the largest field enhancements.

While the reproducible formation of "real" particle pairs or clusters with controlled subnanometer spacing remains a challenging task, the interaction between a NP and its effective image formed in a nearby conducting film (Figure $1 \mathrm{~A})$ provides a conceptually orthogonal approach to generate a well-defined enhancement region. The spacing between a NP and its image can be controlled by the self-assembly of an insulating, nanoscale thin film onto the metal layer. Metal NPs deposited onto the film can thus have a fixed and highly uniform spacing relative to the metal surface and hence to their images. The motivation behind this study is to clarify the contribution of the gap-dependent enhancement region to SERS, which has recently been observed from NPs coated with Raman active molecules and deposited on metal films. ${ }^{33-37}$

To illustrate the efficacy of the NP-film system in generating large field enhancements, we analyze the surfaceenhanced resonant Raman scattering (SERRS) from dyecoated NPs. Using a fluorescent molecule enables single NP SERRS spectra to be acquired, since the electronic resonance associated with the absorption peak provides a scattering cross-section enhancement of many orders of magnitude in addition to the electromagnetic enhancement. The resonant scattering from dye molecules is sufficiently strong that SERRS spectra are obtainable from just a few or even single molecules; thus, resonant dye molecules can serve as efficient probes of nanoscale enhancement. For the experiments presented here, we used malachite green isothiocy- 
anate (MGITC), a fluorophore whose absorbance peak is in the red portion of the visible spectrum, ${ }^{38}$ roughly overlapping with the plasmon resonance of the gold NP-film system and the excitation wavelength.

The reproducibility of this system is due to every NP at a given NP-film spacer, in principle, generating the same field enhancement in the region of the gap with polarization and other properties identical among all NPs. Figure $1 \mathrm{~B}$ provides an illustration of the method we employ whereby a $60 \mathrm{~nm}$ MGITC-coated gold NP is separated from a $45 \mathrm{~nm}$ gold film using a single self-assembled molecular layer of poly(allylamine hydrochloride) (PAH, $\sim 0.6 \mathrm{~nm}$ ). When viewed through a microscope (0.9 NA 100 $\times$ objective, Figure 1C, left), elastic scattering from film-coupled NPs produce "doughnut"shaped point-spread-functions (PSFs) due to the cancellation of the horizontal dipolar response of the NPs and their images. ${ }^{39}$ The uniformity of the PSFs from a collection of NPs within a field of view, as well as the similarity in their intensities and color, provides an indication that the spacing from the film is similar for all NPs. Further confirming the posited orientation of the gap region and the polarized nature of the resonance, it is found that both the elastic scattering and the SERRS intensity from the NP-film system do not respond in any significant way to S-polarized excitation. ${ }^{38}$

The NP-film resonance can be driven either by an incident beam brought in from above the substrate in darkfield illumination (DF: incidence angle $75^{\circ}$ from normal); or from below the substrate, using the total internal reflection (TIR: $45^{\circ}$ ) occurring at the interface to the glass slide substrate. ${ }^{38}$ Laser scattering from NPs and SERRS emission from the MGITC molecules within the NP-film gaps was collected by an optical microscope. When the laser-scattering image (Figure 1C, left) is compared to the SERRS image (Figure 1C, right), we see that every NP-film gap acts as an efficient and localized transmitter of SERRS from MGITC into the far field. Also, in most cases the doughnut-shaped PSF is observed from the single NP-film SERRS, indicating transmission through the vertically oriented dipole. Figure 1D shows a representative SERRS spectrum acquired from a single filmcoupled NP, selected using an adjustable image plane pinhole aperture and relayed to a spectrometer. Distinct resonances corresponding to a collection of vibrational modes specific to the MGITC molecule can be readily identified. ${ }^{40}$

The qualitative understanding of the field-enhancing filmcoupled NP system provided by an image dipole model is complicated by the imperfection of the image. Therefore, a three-dimensional model using COMSOL Multiphysics, a commercial finite-element mode solver, was constructed to gain a more quantitative understanding of the NP-film system and to enable parametric studies. The exact material properties of the NP, film, spacer layer, and the excitation of surface plasmons in the film were incorporated into a full wave simulation model, which enabled visualization of the near-field patterns, collection of the far-field scattered light, and generation of quantitative values for the expected enhancement factors and resonance frequency shifts as a function of parameters of interest, such as incident illumination conditions and NP-film gap distances.

The intensity and color of DF images of elastically scattered white light from a gold NP spaced from a gold film by layer-by-layer deposition of polyelectrolyte (PE) spacer lay$\mathrm{ers}^{38}$ (Figure 2A) correlates well with the simulated spectral shift from the model (Figure 2C) and also previously reported $^{39,41-43}$ blue shift in the plasmon resonance with increased NP-film spacer distance. A more detailed comparison of the simulated scattering curves with both the measured extinction curves and the measured single NP scattering curves are included in the Supporting Information.

The calculated spatial distribution of the field enhancement at the $633 \mathrm{~nm}$ excitation wavelength in the gap region between the NP and its image is shown in Figure 2B. A tighter confinement of the fields within the gap as well as a much greater enhancement of the fields at smaller NP-film gap distances is predicted. At the $1.7 \mathrm{~nm}$ gap distance, the volume of the enhancement region is calculated to be $\sim 1$ $\mathrm{nm}^{3}$, which would fit up to 5 MGITC molecules. ${ }^{38}$ While the majority of signal is likely to originate from just a few molecules, to estimate the total SERRS enhancement the $\mathrm{E}^{4}$ field occupying the region within a shell of $2 \mathrm{~nm}$ thickness around the surface of the spherical NP is averaged and normalized by the incident field. The $2 \mathrm{~nm}$ shell is selected to completely include the $\sim 1 \mathrm{~nm}$ thickness of a monolayer of MGITC molecules on a gold surface. ${ }^{44}$ The calculated values, plotted on a log scale in Figure 2D, show a nonlinear increase with decreasing NP-film gap distance. As the gap narrows, the SERRS signal should climb dramatically.

Experimentally, MGITC SERRS was measured as a function of the NP-film gap dimension by statistically analyzing the total SERRS emission (using DF excitation ${ }^{38}$ ) from single NPs at each PE spacer distance. The integrated CCD intensities of $20 \mathrm{NPs}$ for each sample were averaged and plotted against NP-film gap distance (Figure 3A). Single diffraction limited scatterers were selected for analysis to eliminate any potential enhanced field contributions from aggregated NPs. The averaged SERRS intensities exhibit a trend as a function of the gap distance that is in good agreement with simulated near-field values. The increased variance of the SERRS intensity at higher field values is attributed primarily to deviations in the physical position of each NP on the polyelectrolyte film and fluctuations in the number of MGITC molecules in the field-enhanced region. Slight variations in either will have a significant effect on the enhancement when the gap is on the order of a few nanometers or smaller.

SERRS spectra and SERRS images from single NPs at each PE layer thickness using P-polarized TIR excitation is shown in Figure 3B. Both MGITC SERRS intensity and spectral resolution are highest at the smallest NP-film gap dimension, highlighting the importance of the control over gap distance for maximizing SERRS signal and information content. 

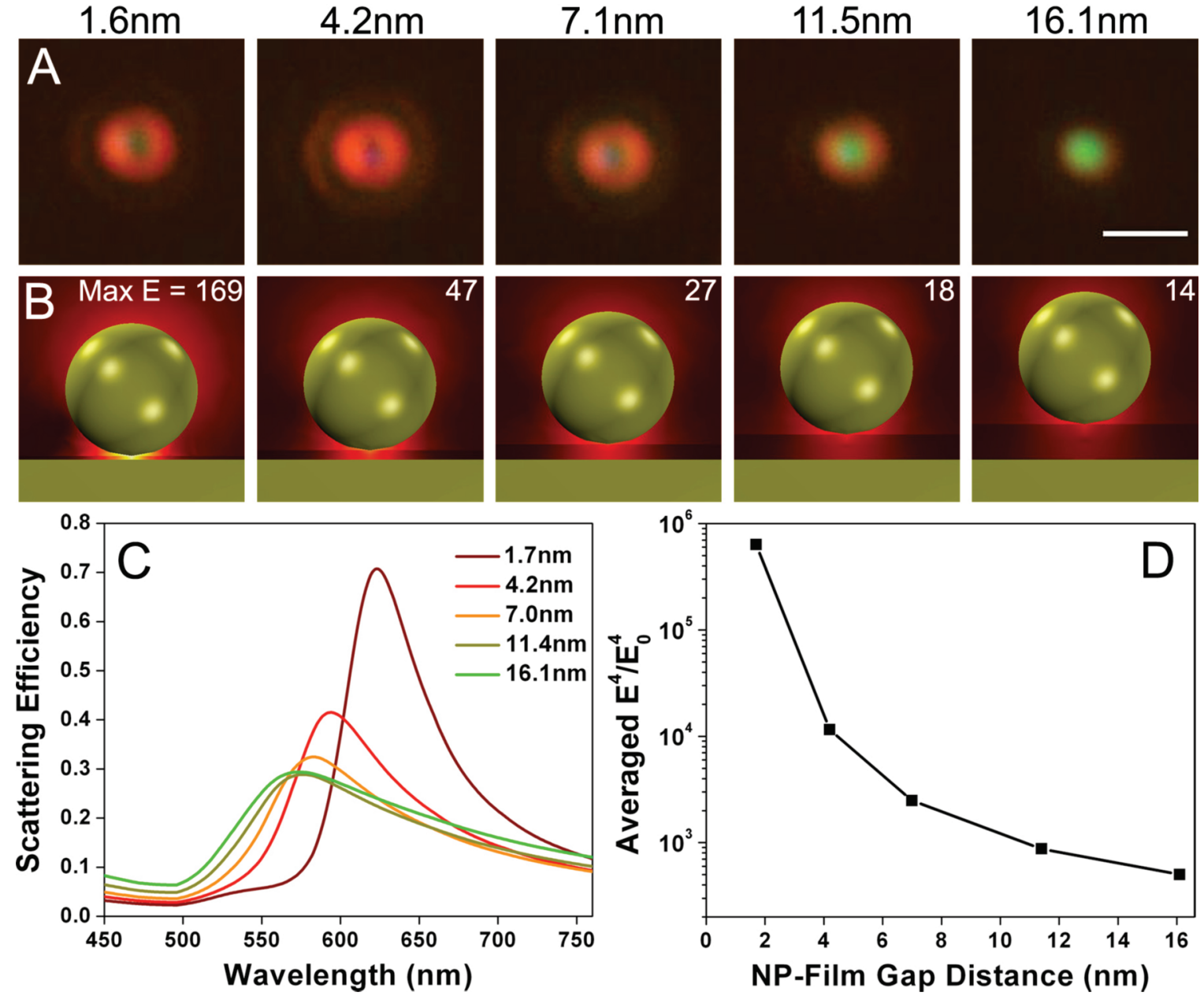

FIGURE 2. (A) Images of elastic scattering from NPs (DF white light illumination) separated from gold support film by increasing PE spacer distances (scale bar $=1 \mu \mathrm{m}$ ). (B) Calculated spatial distribution of the E field (DF illumination, $633 \mathrm{~nm}$ ) at various gap distances where Max $\mathrm{E}$ is the numerical value representing the highest field in each image. (C) Calculated scattering efficiency (DF, scattering cross-section integrated over $\mathrm{NA}=0.9)$. (D) Averaged $\mathrm{E}^{4} / \mathrm{E}_{0}^{4}(\mathrm{DF}, 633 \mathrm{~nm})$ at various gap distances where $\mathrm{E}_{0}$ is the amplitude of the incident beam.
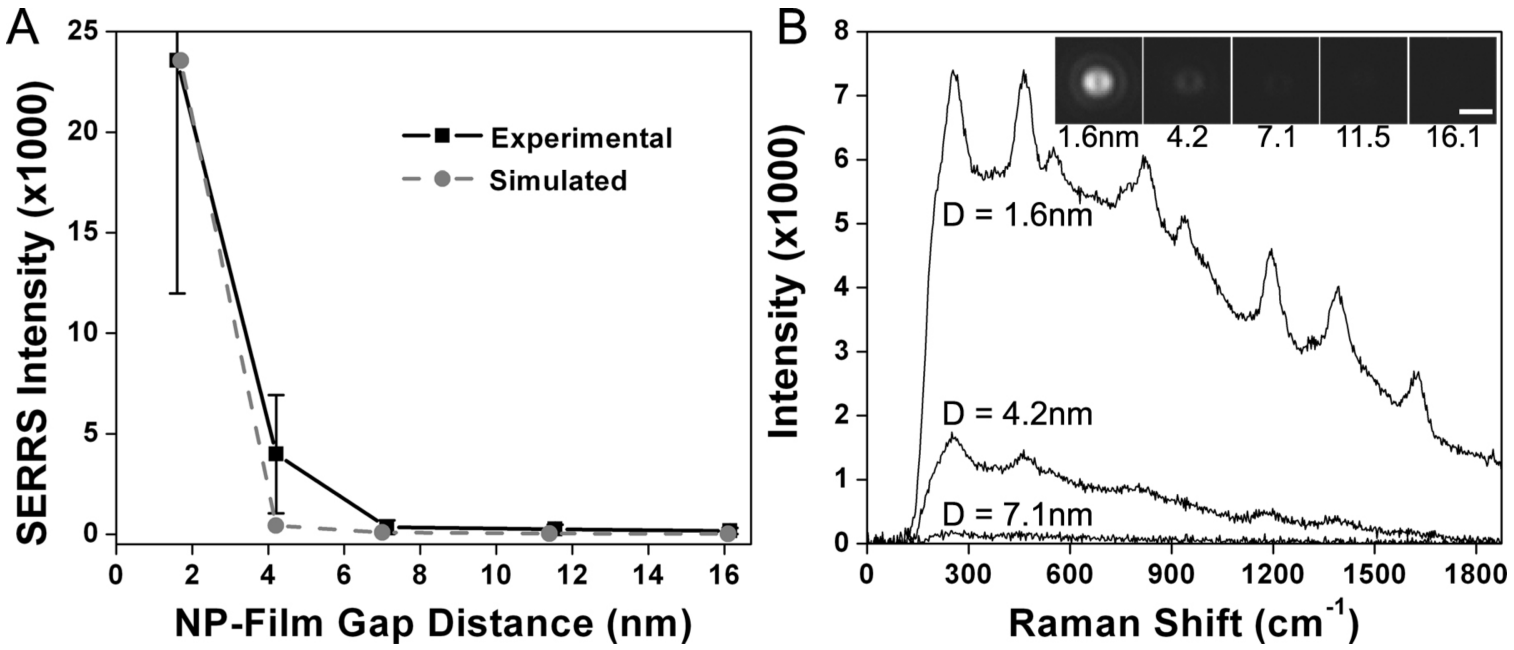

FIGURE 3. Measured spacer dependence of SERRS from MGITC molecules in the NP-film gaps. (A) Averaged SERRS intensities (DF, $633 \mathrm{~nm}$ excitation) from 20 NPs (black squares) compared with normalized values of the field enhancement predicted by simulations (data from Figure 2D, plotted again here in A, gray circles). (B) Representative MGITC SERRS spectra (TIR, $633 \mathrm{~nm}$ excitation) and inset: MGITC SERRS images (scale bar $=1 \mu \mathrm{m}$ ) from single film coupled NPs at increasing gap distance. 
In conclusion, the single particle measurements and simulations presented in this report illustrate the sensitivity of the scattering properties and SERRS enhancements associated with the precise positioning of plasmonic structures. As just one example that demonstrates the value in defining the subnanometer architecture of plasmonic NP systems by a simple and tractable approach, reproducible control over the enhancement regions provides a mechanism for generating high-yield SERRS substrates with consistent field enhancements. Such techniques will be necessary to successfully transition the exciting and sometimes exotic optical phenomena associated with metals to practical plasmonic or metamaterial devices and components.

Acknowledgment. This work was partially supported by NIH Grant R21EB009862. R.T.H. acknowledges support from Award Number F32EB009299 from the NIH NIBIB. D.R.S. and J.J.M. also acknowledge partial support from the Air Force Office of Scientific Research (Contract No. FA9550-09-10562). Aloyse Degiron provided support with simulations. Greg J. Nusz provided MATLAB code for finding centroids of plasmon resonance peaks. Professor John Nolan provided support with measuring bulk Raman spectra. Content is solely the responsibility of the authors and does not represent the official views of the funding agencies.

Supporting Information Available. Description of experimental methods, further discussion of key points in the manuscript, and Supporting Figures S1-S4 are provided. This material is available free of charge via the Internet at http://pubs.acs.org.

\section{REFERENCES AND NOTES}

(1) Gunnarsson, L.; Rindzevicius, T.; Prikulis, J.; Kasemo, B.; Käll, M.; Zou, S.; Schatz, G. J. Phys. Chem. B 2005, 109, 1079-1087.

(2) Mock, J. J.; Barbic, M.; Smith, D. R.; Schultz, D.; Schultz, S. J. Chem Phys. 2002, 116, 6755 .

(3) Orendorff, C.; Sau, T.; Murphy, C. Small 2006, 2, 636-639.

(4) Sönnichsen, C.; Franzl, T.; Wilk, T.; von Plessen, G.; Feldmann, J. New J. Phys. 2002, 4, 93.9193.98.

(5) Su, K.-H.; Wei, Q.-H.; Zhang, X.; Mock, J. J.; Smith, D. R.; Schultz, S. Nano Lett. 2003, 3, 1087-1090.

(6) Sundaramurthy, A.; Crozier, K.; Kino, G.; Fromm, D.; Schuck, P.; Moerner, W. Phys. Rev. B 2005, 72, 165409

(7) Bakker, R.; Drachev, V.; Liu, Z.; Yuan, H.-K.; Pedersen, R.; Boltasseva, A.; Chen, J.; Irudayaraj, J.; Kildishev, A.; Shalaev, V. New J. Phys. 2008, 10, 125022

(8) Schmelzeisen, M.; Zhao, Y.; Klapper, M.; Müllen, K.; Kreiter, M. ACS Nano 2010, 4, 3309-3317.

(9) Fan, J. A.; Wu, C.; Bao, K.; Bao, J.; Bardhan, R.; Halas, N. J.; Manoharan, V. N.; Nordlander, P.; Shvets, G.; Capasso, F. Science 2010, 328, 1135-1138.

(10) Shalaev, V.; Cai, W.; Chettiar, U.; Yuan, H.-K.; Sarychev, A.; Drachev, V.; Kildishev, A. Opt. Lett. 2005, 30, 3356-3358.

(11) Engheta, N. Science 2007, 317, 1698-1702.
(12) Noginov, M. A.; Zhu, G.; Belgrave, A. M.; Bakker, R.; Shalaev, V.; Narimanov, E. E.; Stout, S.; Herz, E.; Suteewong, T.; Wiesner, U. Nature 2009, 460, 1110-1112.

(13) Oulton, R. F.; Sorger, V. J.; Zentgraf, T.; Ma, R.-M.; Gladden, C.; Dai, L.; Bartal, G.; Zhang, X. Nature 2009, 461, 629-632.

(14) Bergman, D.; Stockman, M. Phys. Rev. Lett. 2003, 90, No. 027402

(15) Cubukcu, E.; Kort, E.; Crozier, K.; Capasso, F. Appl. Phys. Lett. 2006, 89, No. 093120

(16) Grady, N. K.; Knight, M. W.; Bardhan, R.; Halas, N. J. Nano Lett. $2010,10,1522-1528$

(17) Palomba, S.; Danckwerts, M.; Novotny, L. J. Opt. A: Pure Appl. Opt. 2009, 11, 114030.

(18) Dionne, J. A.; Diest, K.; Sweatlock, L. A.; Atwater, H. A. Nano Lett. 2009, 9, 897-902

(19) Kottmann, J.; Martin, O.; Smith, D.; Schultz, S. Chem. Phys. Lett. 2001, 341, 1-6.

(20) Garcia-Vidal, F.; Pendry, J. Phys. Rev. Lett. 1996, 77, 1163-1166.

(21) Rycenga, M.; Camargo, P. H. C.; Li, W.; Moran, C. H.; Xia, Y.J. Phys. Chem. Lett. 2010, 1, 696-703.

(22) Aubry, A.; Lei, D. Y.; Fernández-Domínguez, A. I.; Sonnefraud, Y.; Maier, S. A.; Pendry, J. B. Nano Lett. 2010, 10, 2574-2579.

(23) Xu, H.; Aizpurua, J.; Käll, M.; Apell, P. Phys. Rev. E 2000, 62, 43184324.

(24) Imura, K.; Okamoto, H.; Hossain, M.; Kitajima, M. Nano Lett. 2006, 6, 2173-2176.

(25) Xu, H.; Käll, M. ChemPhysChem 2003, 4, 1001-1005.

(26) Gunnarsson, L.; Bjerneld, E.; Xu, H.; Petronis, S.; Kasemo, B.; Käll, M. Appl. Phys. Lett. 2001, 78, 802-804.

(27) Haynes, C.; Van Duyne, R. J. Phys. Chem. B 2003, 107, 74267433.

(28) Su, K.-H.; Durant, S.; Steele, J.; Xiong, Y.; Sun, C.; Zhang, X. J. Phys. Chem. B 2006, 110, 3964-3968.

(29) Chen, S.-Y.; Lazarides, A. A. J. Phys. Chem. C 2009, 113, $12167-$ 12175.

(30) Lim, D.-K.; Jeon, K.-S.; Kim, H. M.; Nam, J.-M.; Suh, Y. D. Nat. Mater. 2010, 9, 60-67.

(31) Vlcková, B.; Moskovits, M.; Pavel, I.; Sisková, K.; Sládková, M.; Slouf, M. Chem. Phys. Lett. 2008, 455, 131-134.

(32) Sun, M.; Fang, Y.; Yang, Z.; Xu, H. Phys. Chem. Chem. Phys. 2009, 11,9412

(33) Anderson, D. J.; Moskovits, M. J. Phys. Chem. B 2006, 110, 1372213727.

(34) Daniels, J. K.; Chumanov, G. J. Phys. Chem. B 2005, 109, 1793617942.

(35) Driskell, J. D.; Lipert, R. J.; Porter, M. D. J. Phys. Chem. B 2006 , 110, 17444-17451.

(36) Orendorff, C. J.; Gole, A.; Sau, T. K.; Murphy, C. J. Anal. Chem. 2005, 77, 3261-3266

(37) Park, W.-H.; Ahn, S.-H.; Kim, Z. H. ChemPhysChem 2008, 9, 2491 2494.

(38) See Supporting Information for descriptions of experimental methods and for supporting discussion

(39) Mock, J. J.; Hill, R. T.; Degiron, A.; Zauscher, S.; Chilkoti, A.; Smith, D. R. Nano Lett. 2008, 8, 2245-2252.

(40) Nguyen, C. T.; Nguyen, J. T.; Rutledge, S.; Zhang, J.; Wang, C.; Walker, G. C. Cancer Lett. 2010, 292, 91-97.

(41) Hu, M.; Ghoshal, A.; Marquez, M.; Kik, P. J. Phys. Chem. C 2010, $114,7509-7514$

(42) Knight, M. W.; Wu, Y.; Lassiter, J. B.; Nordlander, P.; Halas, N. J. Nano Lett. 2009, 9, 2188-2192.

(43) Lévêque, G.; Martin, O. J. F. Opt. Express 2006, 14, 9971-9981.

(44) Domke, K.; Zhang, D.; Pettinger, B. J. Am. Chem. Soc. 2006, 128, $14721-14727$ 